\title{
THE EFFECTS OF EXERCISE ON CARDIOVASCULAR AND RENAL FUNCTION IN CARDIAC PATIENTS WITH AND WITHOUT HEART FAILURE ${ }^{1}$
}

\author{
By WALTER E. JUDSON, WILLIAM HOLLANDER, ${ }^{2}$ J. D. HATCHER, ${ }^{3}$ aND \\ MEYER H. HALPERIN \\ (From the Evans Memorial, Massachusetts Memorial Hospitals, and the Department of \\ Medicine, Boston University School of Medicine, Boston, Mass.)
}

(Submitted for publication March 22, 1955; accepted June 22, 1955)

Exercise in the upright position results in the retention of salt and water in normal subjects (1-5) and in patients with congestive failure (6). The mechanisms which set off these antidiuretic and antisaluretic responses are not definitely known. The following factors have been considered: 1) Sensory stimuli from the legs ; 2) upright position; 3) hemoconcentration; 4) reduction and/or redistribution of the effective circulating blood volume; 5 ) metabolic products or alteration of acid-base balance; and 6) a relatively inadequate cardiac output resulting in a tendency to hypotension or collapse. Studies in this laboratory on normal subjects in the supine position showed that strenuous exercise (alternate straight leg-raising) does not regularly produce such antidiuretic and antisaluretic responses (7). In the present investigation the study was extended to include patients with valvular and nonvalvular forms of heart disease with or without congestive failure, and patients with chronic pulmonary disease with or without congestive failure, to determine: 1) Whether exercise in the supine position in these patients causes antidiuretic and antisaluretic responses; and 2) whether there is a consistent relationship between the cardiodynamic and the renal responses to such exercise.

\section{PATIENTS AND METHODS}

Group A consisted of fifteen patients with severe valvular and non-valvular heart disease. All patients in

\footnotetext{
1 This investigation was supported in part by a grant from the National Heart Institute of the National Institutes of Health, U.S.P.H.S.

2 Formerly United States Public Health Service Fellow in Medicine, Evans Memorial, Massachusetts Memorial Hospitals.

${ }^{3}$ Formerly Fellow in Medicine, Evans Memorial, Massachusetts Memorial Hospitals; Present Address: Queen's University, Kingston, Ontario, Canada.
}

this group were considered to have some degree of circulatory failure as indicated by an impaired cardiac output response during exercise, by an abnormal right ventricular end diastolic pressure of over $8 \mathrm{~mm}$. $\mathrm{Hg}$, or both. Nine of the twelve patients with rheumatic heart disease (mitral stenosis and/or mitral insufficiency and minimal aortic disease) had clinical and cardiodynamic evidence of right ventricular failure. Two patients with arteriosclerotic heart disease and one patient with congenital heart disease and associated pulmonary vascular disease had congestive failure. Five of the patients (A. L., C. S., C. J., F. A., J. C.) had minimal to moderate peripheral edema. All patients with right ventricular failure and/or auricular fibrillation were on maintenance oral digitalis therapy and in some cases were receiving mercurial diuretics.

Group B consisted of eight patients with chronic pulmonary disease. Three of these patients (W. D., M. A., W. H.) had classical evidence of right ventricular hypertrophy by electrocardiogram and roentgenogram of the heart as well as hemodynamic evidence of right heart failure. In three others (M. M., D. K., C. Di.) electrocardiographic or roentgenographic evidence (or both) of right ventricular enlargement was lacking, but the hemodynamic measurements of right ventricular filling pressure indicated mild right ventricular failure, particularly during exercise. The remaining two patients (J. M., M. G.) had pulmonary arterial pressures which were elevated at rest or rose during exercise, but no evidence of congestive failure.

The patients were studied in the post-absorptive state without sedative medication. The experimental procedure and methods of measurement of cardiovascular and renal functions were similar to those previously described (8).

\section{RESULTS}

Individual cardiovascular and renal data at rest and in response to exercise are presented in Tables I and II; detailed data on seven other patients (A. L., C. S., E. M., C. M., E. T., A. C., J. C.) with predominately mitral stenosis have already been published (8). The means for all (including these seven) patients together with a statistical analysis are shown in Table III. 


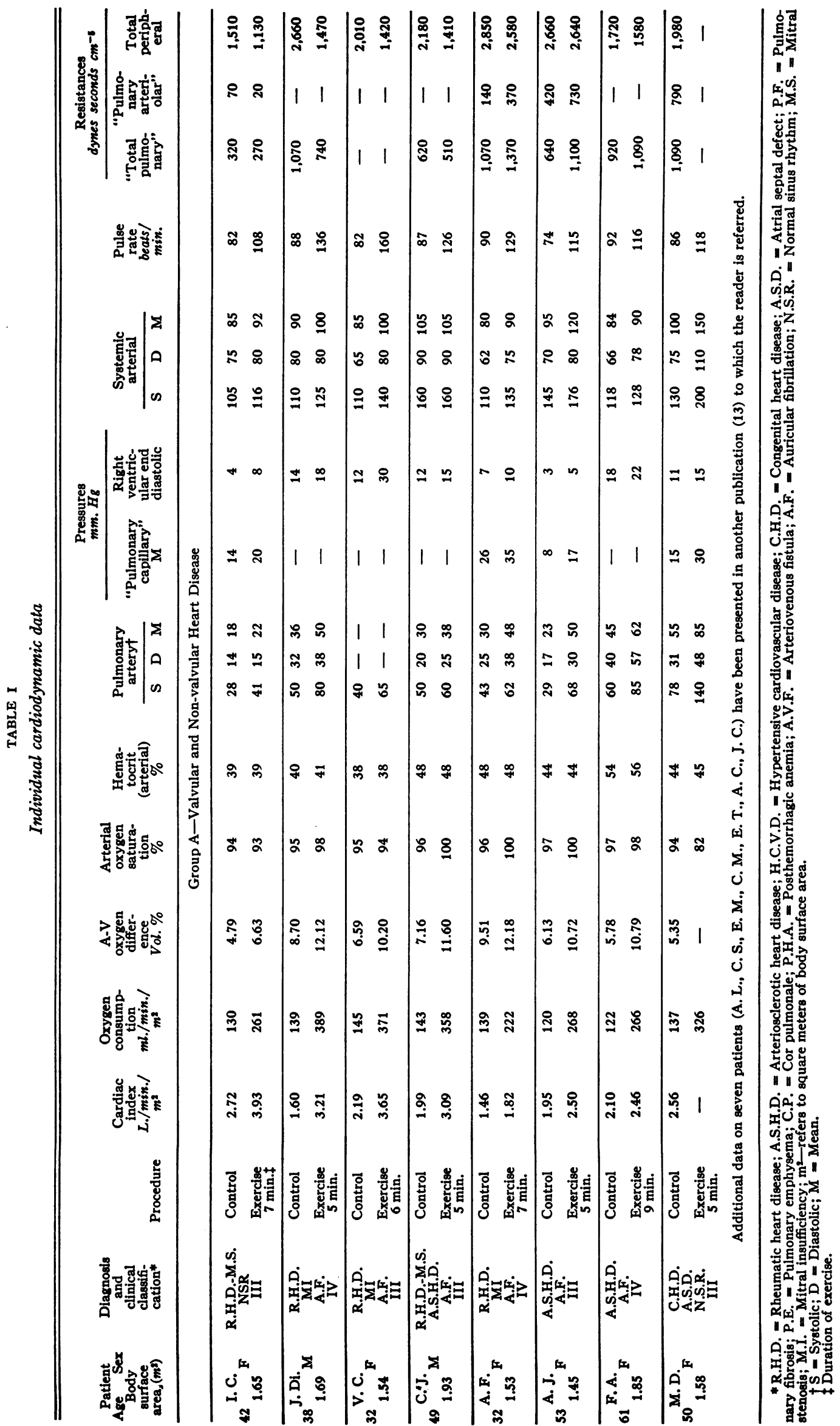




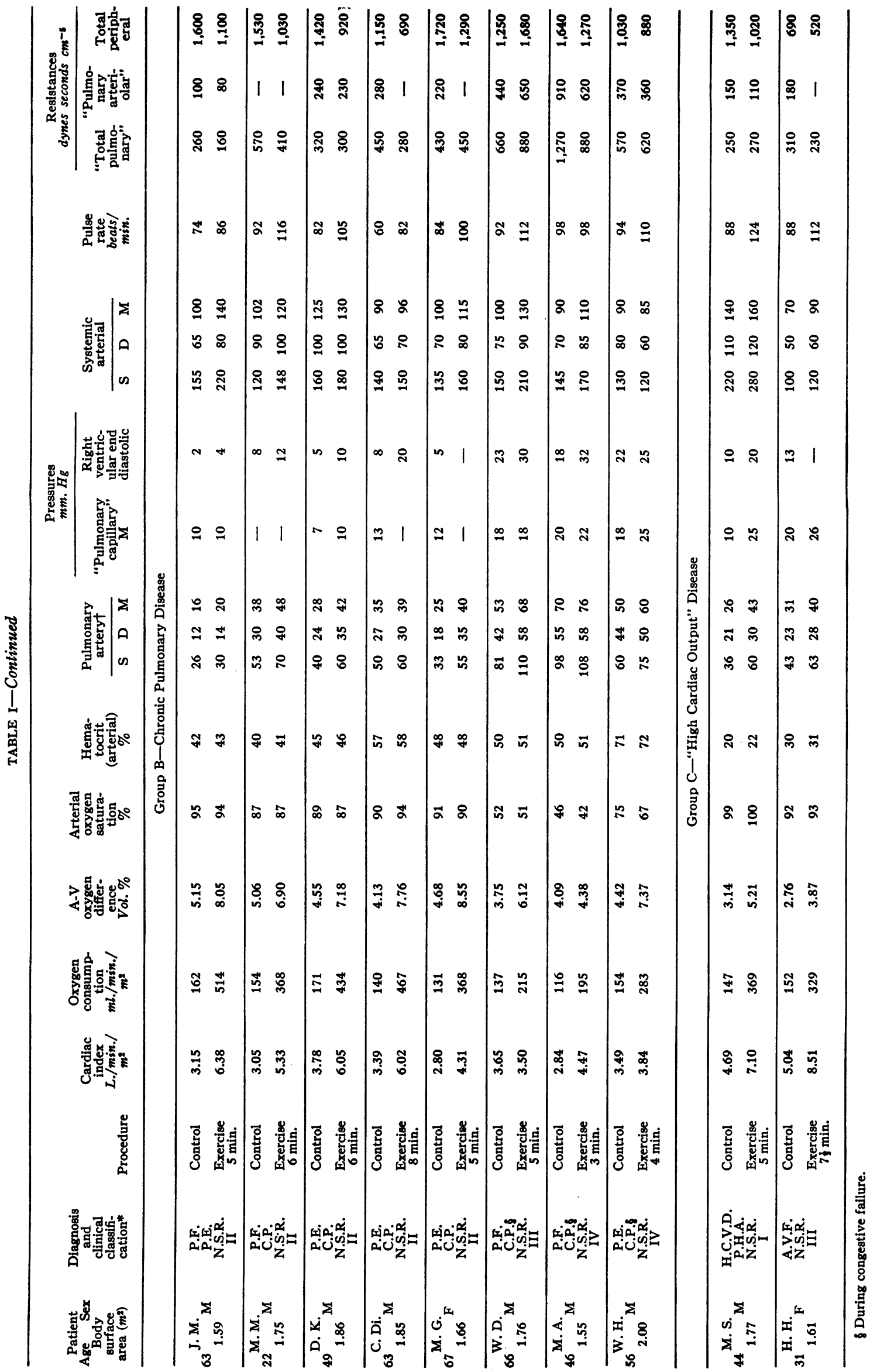




\section{Cardiovascular responses}

Cardiac index and oxygen measurements. At rest the mean cardiac index for the patients with valvular and non-valvular heart disease (Group A) was $2.46 \mathrm{~L}$. per min. per $\mathrm{m}^{2}$. Eleven of the fifteen patients in this group had abnormally low cardiac indices $\left(<2.75 \mathrm{~L}\right.$. per min. per $\left.\mathrm{m}^{2}\right)$. In contrast, all of the patients with chronic pulmonary disease with or without congestive failure (Group B) had normal cardiac indices at rest, the average being $3.27 \mathrm{~L}$. per min. per $\mathrm{m}^{2}$. The mean difference of $0.81 \mathrm{~L}$. per min. per $\mathrm{m}^{2}$ between the resting cardiac indices of these two groups was highly significant statistically. However, in response to exercise the mean increase in cardiac index for both groups was of similar magnitude and repre- sented a highly significant change (average $1.08 \mathrm{~L}$. per min. per $\mathrm{m}^{2}$ or 44 per cent for Group $A$ and 1.72 L. per min. per $\mathrm{m}^{2}$ or 53 per cent for Group B). Although the response of the cardiac index to exercise in Group B was $0.63 \mathrm{~L}$. per min. per $\mathrm{m}^{2}$ greater, this difference was not statistically significant.

Oxygen consumption at rest and in response to exercise was similar in the two groups. This suggested that the two groups performed comparable amounts of work during exercise. The mean arteriovenous oxygen difference in Group A was abnormally elevated at rest (mean 6.01 vol. per cent) and during exercise (10.14 vol. per cent). On the other hand, the mean arteriovenous oxygen difference in Group B was within the normal

TABLE II

Individual renal data

\begin{tabular}{|c|c|c|c|c|c|c|c|c|c|c|}
\hline Patient & $\begin{array}{l}\text { Diagnosis } \\
\text { and clinical } \\
\text { classification }\end{array}$ & Procedure & Periods & $\underset{\substack{\text { ml. } / \min . / \\
1.73 \mathrm{~m}^{2}}}{\text { PaH }}$ & $\underset{\substack{\operatorname{ml} . / \min . / \\
1.73 \mathrm{~m}^{2}}}{\mathrm{C}_{\mathrm{N}}}$ & F.F. & $\underset{\text { UV. }}{\text { min. }}$ & $\underset{\substack{\mathrm{UNaV}_{\mathrm{Na}} \\
\text { micro. }}}{\min . /}$ & $\underset{\substack{\text { UicroElV } \\
\text { min. }}}{\text { mat }}$ & $\underset{\substack{\mathrm{UKV}_{\mathrm{KV}} \\
\text { microEq. } \\
\min .}}{ }$ \\
\hline \multicolumn{11}{|c|}{ Group A-Valvular and Non-valvular Heart Disease } \\
\hline \multirow[t]{2}{*}{ I. C. } & \multirow{2}{*}{$\begin{array}{l}\text { R.H.D. } \\
\text { M.S. } \\
\text { N.S.R. } \\
\text { III }\end{array}$} & \multirow{2}{*}{$\begin{array}{l}\text { Control } \\
\text { Exercise } \\
7 \text { min. } \\
\text { Recovery }\end{array}$} & $\frac{1-4}{5}$ & $\begin{array}{l}350 \\
215\end{array}$ & $\begin{array}{l}93 \\
58\end{array}$ & $\begin{array}{l}27 \\
27\end{array}$ & $\begin{array}{l}5.8 \\
1.3\end{array}$ & $\begin{array}{l}24 \\
11\end{array}$ & $\begin{array}{l}24 \\
17\end{array}$ & $\begin{array}{l}24 \\
19\end{array}$ \\
\hline & & & $6-7$ & 248 & 75 & 30 & 0.8 & 12 & 16 & 27 \\
\hline \multirow[t]{2}{*}{ J. D. } & \multirow{2}{*}{$\begin{array}{l}\text { R.H.D. } \\
\text { M.I. } \\
\text { A.F. } \\
\text { IV }\end{array}$} & \multirow{2}{*}{$\begin{array}{l}\text { Control } \\
\text { Exercise } \\
5 \text { min. } \\
\text { Recovery }\end{array}$} & $\begin{array}{c}1-3 \\
4\end{array}$ & $\begin{array}{l}206 \\
106\end{array}$ & $\begin{array}{l}94 \\
44\end{array}$ & $\begin{array}{l}45 \\
41\end{array}$ & $\begin{array}{l}2.1 \\
1.7\end{array}$ & $\begin{array}{r}132 \\
66\end{array}$ & $\begin{array}{r}144 \\
78\end{array}$ & $\begin{array}{r}109 \\
53\end{array}$ \\
\hline & & & $5-6$ & 208 & 101 & 49 & 0.6 & 30 & 63 & 79 \\
\hline \multirow[t]{2}{*}{ V. C. } & \multirow{2}{*}{$\begin{array}{l}\text { R.H.D. } \\
\text { M.I. } \\
\text { A.F. } \\
\text { III }\end{array}$} & \multirow{2}{*}{$\begin{array}{l}\text { Control } \\
\text { Exercise } \\
6 \text { min. } \\
\text { Recovery }\end{array}$} & $1 \frac{-4}{5}$ & $\begin{array}{l}477 \\
207\end{array}$ & & & $\begin{array}{l}6.2 \\
6.7\end{array}$ & $\begin{array}{l}22 \\
18\end{array}$ & $\begin{array}{l}42 \\
30\end{array}$ & $\begin{array}{l}53 \\
25\end{array}$ \\
\hline & & & $6-8$ & 343 & & & 3.3 & 16 & 33 & 32 \\
\hline \multirow[t]{2}{*}{ C. J.* } & \multirow{2}{*}{$\begin{array}{l}\text { R.H.D. } \\
\text { M.S. } \\
\text { A.S.H.D. } \\
\text { A.F. } \\
\text { III }\end{array}$} & \multirow{2}{*}{$\begin{array}{l}\text { Control } \\
\text { Exercise } \\
5 \text { min. } \\
\text { Recovery }\end{array}$} & $\begin{array}{c}1-3 \\
4\end{array}$ & $\begin{array}{l}279 \\
332\end{array}$ & $\begin{array}{l}104 \\
123\end{array}$ & $\begin{array}{l}37 \\
40\end{array}$ & $\begin{array}{l}0.7 \\
0.7\end{array}$ & $\begin{array}{l}32 \\
20\end{array}$ & $\begin{array}{l}39 \\
40\end{array}$ & $\begin{array}{l}36 \\
45\end{array}$ \\
\hline & & & $5-6$ & 246 & 106 & 43 & 0.9 & 24 & 36 & 52 \\
\hline \multirow[t]{2}{*}{ A. F. } & \multirow{2}{*}{$\begin{array}{l}\text { R.H.D. } \\
\text { M.I. } \\
\text { A.F. } \\
\text { IV }\end{array}$} & \multirow{2}{*}{$\begin{array}{l}\text { Control } \\
\text { Exercise } \\
7 \text { min. } \\
\text { Recovery }\end{array}$} & $1-4$ & $\begin{array}{l}357 \\
268\end{array}$ & & & $\begin{array}{l}6.9 \\
5.9\end{array}$ & $\begin{array}{l}9 \\
7\end{array}$ & $\begin{array}{l}72 \\
56\end{array}$ & $\begin{array}{l}21 \\
21\end{array}$ \\
\hline & & & $6-8$ & 289 & & & 5.8 & 8 & 53 & 21 \\
\hline \multirow[t]{2}{*}{ A. J. } & \multirow[t]{2}{*}{$\begin{array}{l}\text { A.S.H.D. } \\
\text { A.F. } \\
\text { III }\end{array}$} & \multirow{2}{*}{$\begin{array}{l}\text { Control } \\
\text { Exercise } \\
5 \text { min. } \\
\text { Recovery }\end{array}$} & $\begin{array}{c}1-5 \\
6\end{array}$ & $\begin{array}{l}287 \\
268\end{array}$ & $\begin{array}{l}106 \\
100\end{array}$ & $\begin{array}{l}37 \\
37\end{array}$ & $\begin{array}{l}8.4 \\
0.6\end{array}$ & $\begin{array}{r}196 \\
63\end{array}$ & $\begin{array}{r}130 \\
44\end{array}$ & $\begin{array}{l}55 \\
50\end{array}$ \\
\hline & & & $7-8$ & 295 & 98 & 33 & 0.5 & 49 & 42 & 60 \\
\hline \multirow[t]{2}{*}{ F.A. } & \multirow[t]{2}{*}{$\begin{array}{l}\text { A.S.H.D. } \\
\text { A.F. } \\
\text { IV }\end{array}$} & \multirow{2}{*}{$\begin{array}{l}\text { Control } \\
\text { Exercise } \\
9 \text { min. } \\
\text { Recovery } \\
\end{array}$} & $\begin{array}{c}1-3 \\
4\end{array}$ & $\begin{array}{l}143 \\
130\end{array}$ & $\begin{array}{l}56 \\
46\end{array}$ & $\begin{array}{l}39 \\
36\end{array}$ & $\begin{array}{l}0.8 \\
0.4\end{array}$ & $\begin{array}{l}35 \\
22\end{array}$ & $\begin{array}{l}58 \\
44\end{array}$ & $\begin{array}{l}43 \\
40\end{array}$ \\
\hline & & & $5-6$ & 151 & 60 & 40 & 0.5 & 30 & 53 & 51 \\
\hline \multirow[t]{2}{*}{ M. D. } & \multirow{2}{*}{$\begin{array}{l}\text { C.H.D. } \\
\text { I.A.S.D. } \\
\text { N.S.R. } \\
\text { III }\end{array}$} & \multirow{2}{*}{$\begin{array}{l}\text { Control } \\
\text { Exercise } \\
5 \text { min. } \\
\text { Recovery }\end{array}$} & $1-4$ & $\begin{array}{l}193 \\
143\end{array}$ & $\begin{array}{l}89 \\
65\end{array}$ & $\begin{array}{l}46 \\
45\end{array}$ & $\begin{array}{l}4.9 \\
0.9\end{array}$ & $\begin{array}{l}69 \\
30\end{array}$ & $\begin{array}{l}64 \\
42\end{array}$ & $\begin{array}{l}41 \\
35\end{array}$ \\
\hline & & & 6 & 194 & 81 & 42 & 0.4 & 22 & 19 & 32 \\
\hline
\end{tabular}


TABLE n-Continued

\begin{tabular}{|c|c|c|c|c|c|c|c|c|c|c|}
\hline Patient & $\begin{array}{c}\text { Diagnosis } \\
\text { and clinical } \\
\text { classification }\end{array}$ & Procedure & Periods & 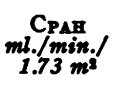 & 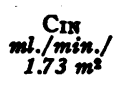 & F.F. & $\underset{\substack{\text { UV } \\
\text { min. }}}{\text {. }}$ & 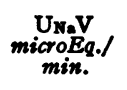 & 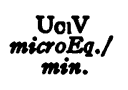 & 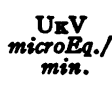 \\
\hline \multicolumn{11}{|c|}{ Group B-Chronic Pulmonary Disease } \\
\hline \multirow[t]{2}{*}{ J. M. } & \multirow{2}{*}{$\begin{array}{l}\text { P.F. } \\
\text { P.E. } \\
\text { N.S.R. } \\
\text { II }\end{array}$} & \multirow{2}{*}{$\begin{array}{l}\text { Control } \\
\text { Exercise } \\
5 \text { min. } \\
\text { Recovery }\end{array}$} & $\frac{1-4}{5}$ & $\begin{array}{l}292 \\
183\end{array}$ & $\begin{array}{l}85 \\
66\end{array}$ & $\begin{array}{l}30 \\
36\end{array}$ & $\begin{array}{l}1.8 \\
0.3\end{array}$ & $\begin{array}{l}84 \\
29\end{array}$ & $\begin{array}{r}128 \\
60\end{array}$ & $\begin{array}{l}46 \\
31\end{array}$ \\
\hline & & & $6-7$ & 272 & 91 & 33 & 0.8 & 57 & 96 & 52 \\
\hline \multirow[t]{2}{*}{ M. M. } & \multirow{2}{*}{$\begin{array}{c}\text { P.F. } \\
\text { C.P. } \\
\text { N.S.R. } \\
\text { II }\end{array}$} & \multirow{2}{*}{$\begin{array}{l}\text { Control } \\
\text { Exercise } \\
6 \text { min. } \\
\text { Recovery }\end{array}$} & $\begin{array}{c}1-3 \\
4\end{array}$ & $\begin{array}{l}266 \\
180\end{array}$ & $\begin{array}{l}84 \\
77\end{array}$ & $\begin{array}{l}31 \\
43\end{array}$ & $\begin{array}{l}2.6 \\
1.6\end{array}$ & $\begin{array}{l}192 \\
166\end{array}$ & & $\begin{array}{l}90 \\
70\end{array}$ \\
\hline & & & $5-6$ & 208 & 91 & 43 & 1.9 & 186 & & 152 \\
\hline \multirow[t]{2}{*}{ D. $\mathrm{K}$. } & \multirow{2}{*}{$\begin{array}{c}\text { P.E. } \\
\text { C.P. } \\
\text { N.S.R. } \\
\text { II }\end{array}$} & \multirow{2}{*}{$\begin{array}{l}\text { Control } \\
\text { Exercise } \\
6 \text { min. } \\
\text { Recovery }\end{array}$} & $1-4$ & $\begin{array}{l}438 \\
433\end{array}$ & $\begin{array}{l}112 \\
126\end{array}$ & $\begin{array}{l}26 \\
29\end{array}$ & $\begin{array}{l}1.8 \\
1.5\end{array}$ & $\begin{array}{l}148 \\
128\end{array}$ & $\begin{array}{l}152 \\
148\end{array}$ & $\begin{array}{l}86 \\
82\end{array}$ \\
\hline & & & $6-7$ & 419 & 115 & 27 & 1.4 & 128 & 132 & 81 \\
\hline \multirow[t]{2}{*}{ C. D. } & \multirow{2}{*}{$\begin{array}{c}\text { P.E. } \\
\text { C.P. } \\
\text { N.S.R. }\end{array}$} & \multirow{2}{*}{$\begin{array}{l}\text { Control } \\
\text { Exercise } \\
8 \text { min. } \\
\text { Recovery }\end{array}$} & $\begin{array}{c}1-3 \\
4\end{array}$ & $\begin{array}{l}347 \\
319\end{array}$ & $\begin{array}{r}92 \\
100\end{array}$ & $\begin{array}{l}26 \\
31\end{array}$ & $\begin{array}{l}0.6 \\
0.8\end{array}$ & $\begin{array}{l}23 \\
20\end{array}$ & $\begin{array}{l}11 \\
14\end{array}$ & $\begin{array}{l}23 \\
24\end{array}$ \\
\hline & & & $5-7$ & 372 & 104 & 27 & 3.0 & 17 & 18 & 41 \\
\hline \multirow[t]{2}{*}{ M. G. } & \multirow{2}{*}{$\begin{array}{l}\text { P.E. } \\
\text { C.P. } \\
\text { N.S.R. } \\
\text { II }\end{array}$} & \multirow{2}{*}{$\begin{array}{l}\text { Control } \\
\text { Exercise } \\
5 \text { min. } \\
\text { Recovery }\end{array}$} & $\begin{array}{c}1-3 \\
4\end{array}$ & $\begin{array}{l}176 \\
173\end{array}$ & $\begin{array}{l}51 \\
51\end{array}$ & $\begin{array}{l}30 \\
30\end{array}$ & $\begin{array}{l}2.1 \\
2.0\end{array}$ & $\begin{array}{l}95 \\
67\end{array}$ & $\begin{array}{l}64 \\
52\end{array}$ & $\begin{array}{l}48 \\
25\end{array}$ \\
\hline & & & $5-7$ & 170 & 51 & 30 & 1.9 & 45 & 35 & 38 \\
\hline \multirow[t]{2}{*}{ W. D. } & \multirow{2}{*}{$\begin{array}{l}\text { P.F. } \\
\text { C.P.* } \\
\text { N.S.R. } \\
\text { III }\end{array}$} & \multirow{2}{*}{$\begin{array}{l}\text { Control } \\
\text { Exercise } \\
5 \text { min. } \\
\text { Recovery }\end{array}$} & $\begin{array}{c}1-3 \\
4\end{array}$ & $\begin{array}{l}212 \\
106\end{array}$ & $\begin{array}{l}91 \\
58\end{array}$ & $\begin{array}{l}43 \\
54\end{array}$ & $\begin{array}{l}1.2 \\
0.6\end{array}$ & $\begin{array}{l}22 \\
27\end{array}$ & $\begin{array}{l}48 \\
40\end{array}$ & $\begin{array}{l}53 \\
28\end{array}$ \\
\hline & & & $5-7$ & 198 & 93 & 50 & 0.4 & 20 & 41 & 48 \\
\hline \multirow[t]{2}{*}{ M. A. } & \multirow{2}{*}{$\begin{array}{l}\text { P.F. } \\
\text { C.P.* } \\
\text { N.S.R. } \\
\text { IV }\end{array}$} & Control & $1-3$ & 349 & & & 1.0 & 241 & 217 & 97 \\
\hline & & $\begin{array}{l}3 \text { min. } \\
\text { Recovery }\end{array}$ & $5-7$ & 332 & & & 1.7 & 161 & 162 & 94 \\
\hline \multirow[t]{2}{*}{ W. H. } & \multirow{2}{*}{$\begin{array}{l}\text { P.E. } \\
\text { C.P.* } \\
\text { N.S.R. } \\
\text { IV }\end{array}$} & \multirow{2}{*}{$\begin{array}{l}\text { Control } \\
\text { Exercise } \\
4 \text { min. } \\
\text { Recovery }\end{array}$} & $1-3$ & 212 & 91 & 43 & 0.7 & 28 & 46 & 51 \\
\hline & & & $5-6$ & 202 & 89 & 44 & 0.3 & 7 & 32 & 45 \\
\hline \multicolumn{11}{|c|}{ Group C-"High-Cardiac-Output" Disease } \\
\hline \multirow[t]{2}{*}{ M. S.† } & \multirow{2}{*}{$\begin{array}{c}\text { H.C.V.D. } \\
\text { P.H.A. } \\
\text { N.S.R. } \\
\text { I }\end{array}$} & \multirow{2}{*}{$\begin{array}{l}\text { Control } \\
\text { Exercise } \\
5 \text { min. } \\
\text { Recovery }\end{array}$} & $\begin{array}{c}1-3 \\
4\end{array}$ & $\begin{array}{l}452 \\
403\end{array}$ & $\begin{array}{r}100 \\
96\end{array}$ & $\begin{array}{l}22 \\
24\end{array}$ & $\begin{array}{r}12.0 \\
7.6\end{array}$ & $\begin{array}{l}890 \\
730\end{array}$ & & $\begin{array}{l}93 \\
78\end{array}$ \\
\hline & & & $5-7$ & 414 & 96 & 23 & 3.7 & 297 & & 62 \\
\hline \multirow[t]{2}{*}{ H. H. } & \multirow{2}{*}{$\begin{array}{l}\text { A.V.F. } \\
\text { N.S.R. } \\
\text { III }\end{array}$} & \multirow{2}{*}{$\begin{array}{c}\text { Control } \\
\text { Exercise } \\
7 \frac{1}{2} \text { min. } \\
\text { Recovery }\end{array}$} & $\begin{array}{c}1-4 \\
5\end{array}$ & $\begin{array}{l}289 \\
230\end{array}$ & $\begin{array}{l}93 \\
76\end{array}$ & $\begin{array}{l}32 \\
33\end{array}$ & $\begin{array}{l}1.4 \\
0.5\end{array}$ & $\begin{array}{r}12 \\
6\end{array}$ & $\begin{array}{r}12 \\
5\end{array}$ & $\begin{array}{l}79 \\
61\end{array}$ \\
\hline & & & $6-8$ & 291 & 98 & 34 & 0.8 & 13 & 5 & 79 \\
\hline
\end{tabular}

* During congestive failure.

† Patient was given $300 \mathrm{ml}$. 5 per cent $\mathrm{NaCl}$ I.V.

range at rest (4.47 vol. per cent) and during exercise (7.04 vol. per cent). The differences between the values of the two groups at rest and during exercise and the change in values in response to exercise were all highly significant statistically. Thus, the increases in cardiac output during exercise in Group A were not commensurate with the associated rises in oxygen consump- tion so abnormally. elevated mixed arteriovenous oxygen differences resulted. In the patients in Group B, however, the increases in cardiac output during exercise were comparable with the associated rises in oxygen consumption so the arteriovenous oxygen differences showed normal increases.

The mean arterial oxygen saturation for Group 
TABLE III

Mean data and statistical analysis

\begin{tabular}{|c|c|c|c|c|c|c|c|c|}
\hline & \multicolumn{4}{|c|}{$\begin{array}{c}\text { Cardiac index } \\
L . / \min . / m^{2}\end{array}$} & \multicolumn{4}{|c|}{$\begin{array}{l}\text { Oxygen consumption } \\
\mathrm{L} . / \mathrm{min} . / \mathrm{m}^{2}\end{array}$} \\
\hline & Rest & Exercise & $\begin{array}{c}\text { Response to } \\
\text { exercise }\end{array}$ & $\mathbf{P}$ & Rest & Exercise & $\begin{array}{c}\text { Response to } \\
\text { exercise }\end{array}$ & $\mathbf{P}$ \\
\hline \multirow[t]{3}{*}{$\begin{array}{l}\text { Group A } \\
\text { Group B } \\
\text { Diff. } \\
\text { P }\end{array}$} & $\begin{array}{c}2.46 \pm 0.14 \\
3.27 \pm 0.10 \\
+0.81 \pm 0.17 \\
<0.01\end{array}$ & $\begin{array}{r}3.56 \pm 0.26 \\
4.98 \pm 0.40 \\
+1.42 \pm 0.47 \\
0.06\end{array}$ & $\begin{array}{c}+1.08 \pm 0.14 \\
+1.72 \pm 0.40 \\
+0.63 \pm 0.42 \\
0.1\end{array}$ & $\begin{array}{c}<0.01 \\
<0.01 \\
- \\
-\end{array}$ & $\begin{array}{c}141 \pm 5 \\
146 \pm 6 \\
+5 \pm 8 \\
0.5\end{array}$ & $\begin{array}{c}348 \pm 20 \\
356 \pm 41 \\
+8 \pm 46 \\
0.9\end{array}$ & $\begin{array}{c}+206 \pm 17 \\
+210 \pm 37 \\
+4 \pm 41 \\
>0.9\end{array}$ & $\begin{array}{c}<0.01 \\
<0.01 \\
- \\
-\end{array}$ \\
\hline & \multicolumn{4}{|c|}{$\begin{array}{l}\text { A-V O2 difference } \\
\text { Vol. } \% \\
\end{array}$} & \multicolumn{4}{|c|}{$\begin{array}{c}\text { Arterial oxygen saturation } \\
\%\end{array}$} \\
\hline & Rest & Exercise & $\begin{array}{l}\text { Response to } \\
\text { exercise }\end{array}$ & $\mathbf{P}$ & Rest & Exercise & $\begin{array}{c}\text { Response to } \\
\text { exercise }\end{array}$ & $\mathbf{P}$ \\
\hline \multirow[t]{4}{*}{$\begin{array}{l}\text { Group A } \\
\text { Group B } \\
\text { Diff. } \\
\text { P }\end{array}$} & $\begin{array}{c}6.01 \pm 0.39 \\
4.47 \pm 0.17 \\
-1.54 \pm 0.42 \\
<0.01\end{array}$ & $\begin{array}{r}10.14 \pm 0.52 \\
7.04 \pm 0.46 \\
-3.10 \pm 0.69 \\
<0.01\end{array}$ & $\begin{array}{c}+4.09 \pm 0.33 \\
+2.56 \pm 0.40 \\
-1.53 \pm 0.51 \\
<0.01\end{array}$ & $\begin{array}{c}<0.01 \\
<0.01 \\
- \\
-\end{array}$ & $\begin{array}{c}95 \pm 3 \\
78 \pm 7 \\
-17 \pm 7 \\
0.03\end{array}$ & $\begin{array}{c}95 \pm 2 \\
76 \pm 7 \\
-19 \pm 8 \\
0.02\end{array}$ & $\begin{array}{c}+0.4 \pm 0.9 \\
-2 \pm 1 \\
-2.4 \pm 1.5 \\
0.2\end{array}$ & $\begin{array}{l}0.7 \\
0.2 \\
- \\
-\end{array}$ \\
\hline & \multirow{2}{*}{\multicolumn{4}{|c|}{$\underset{\%}{\text { Hematocrit }}$}} & \multicolumn{4}{|c|}{ Pressures } \\
\hline & & & & & \multicolumn{4}{|c|}{$\begin{array}{c}\text { Mean pulmonary arterial } \\
\mathbf{m m} . \boldsymbol{H}_{\boldsymbol{g}}\end{array}$} \\
\hline & Rest & Exercise & $\begin{array}{c}\text { Response to } \\
\text { exercise }\end{array}$ & $\mathbf{P}$ & Rest & Exercise & $\begin{array}{c}\text { Response to } \\
\text { exercise }\end{array}$ & $\mathbf{P}$ \\
\hline \multirow[t]{4}{*}{$\begin{array}{l}\text { Group A } \\
\text { Group B } \\
\text { Diff. } \\
\text { P } \\
\end{array}$} & $\begin{array}{c}42 \pm 2 \\
50 \pm 4 \\
+8 \pm 4 \\
0.05\end{array}$ & $\begin{array}{c}44 \pm 1 \\
51 \pm 4 \\
+7 \pm 4 \\
0.09\end{array}$ & $\begin{array}{c}+1 \pm 0.3 \\
+1 \pm 0.1 \\
0 \pm 0.3 \\
0.1\end{array}$ & $\begin{array}{c}<0.01 \\
<0.01 \\
- \\
-\end{array}$ & $\begin{array}{c}38 \pm 4 \\
39 \pm 6 \\
+1 \pm 8 \\
0.9\end{array}$ & $\begin{array}{r}60 \pm 6 \\
49 \pm 6 \\
-11 \pm 8 \\
0.2\end{array}$ & $\begin{array}{c}+22 \pm 3 \\
+10 \pm 2 \\
-12 \pm 4 \\
<0.01\end{array}$ & $\begin{array}{c}<0.01 \\
<0.01 \\
-\end{array}$ \\
\hline & \multicolumn{8}{|c|}{ Pressures } \\
\hline & \multicolumn{4}{|c|}{$\begin{array}{c}\text { Mean "pulmonary capillary" } \\
\mathbf{m m} . \mathbf{H g}\end{array}$} & \multicolumn{4}{|c|}{$\begin{array}{l}\text { Right ventricular end diastolic } \\
m m . H g\end{array}$} \\
\hline & Rest & Exercise & $\begin{array}{l}\text { Response to } \\
\text { exercise }\end{array}$ & $\mathbf{P}$ & Rest & Exercise & $\begin{array}{c}\text { Response to } \\
\text { exercise }\end{array}$ & $\mathbf{P}$ \\
\hline \multirow[t]{4}{*}{$\begin{array}{l}\text { Group A } \\
\text { Group B } \\
\text { Diff. } \\
\text { P }\end{array}$} & $\begin{array}{c}22 \pm 2 \\
14 \pm 2 \\
-8 \pm 3 \\
0.02\end{array}$ & $\begin{array}{r}36 \pm 4 \\
17 \pm 3 \\
-19 \pm 5 \\
<0.01\end{array}$ & $\begin{array}{r}14 \pm 2 \\
+2 \pm 1 \\
-12 \pm 3 \\
<0.01\end{array}$ & $\begin{array}{c}<0.01 \\
0.1 \\
- \\
-\end{array}$ & $\begin{array}{r}9 \pm 1 \\
11 \pm 3 \\
+2 \pm 3 \\
0.6\end{array}$ & $\begin{array}{c}15 \pm 2 \\
19 \pm 4 \\
+4 \pm 5 \\
0.4\end{array}$ & $\begin{array}{c}+6 \pm 1 \\
+7 \pm 2 \\
+1 \pm 2 \\
0.8\end{array}$ & $\begin{array}{r}<0.01 \\
<0.01 \\
- \\
-\end{array}$ \\
\hline & \multicolumn{4}{|c|}{ Pressures } & & & & \\
\hline & \multicolumn{4}{|c|}{$\begin{array}{c}\text { Mean systemic } \\
\mathbf{m m} . \mathbf{H g}\end{array}$} & \multicolumn{4}{|c|}{$\begin{array}{l}\text { Pulse rate } \\
\text { beats/min. }\end{array}$} \\
\hline & Rest & Exercise & $\begin{array}{l}\text { Response to } \\
\text { exercise }\end{array}$ & $\mathbf{P}$ & Rest & Exercise & $\begin{array}{l}\text { Response to } \\
\text { exercise }\end{array}$ & $\mathbf{P}$ \\
\hline \multirow[t]{4}{*}{$\begin{array}{l}\text { Group A } \\
\text { Group B } \\
\text { Diff. } \\
\text { P }\end{array}$} & $\begin{array}{c}95 \pm 3 \\
100 \pm 2 \\
+5 \pm 5 \\
0.3\end{array}$ & $\begin{array}{c}117 \pm 8 \\
116 \pm 6 \\
-1 \pm 10 \\
>0.9\end{array}$ & $\begin{array}{c}+22 \pm 6 \\
+15 \pm 5 \\
-7 \pm 8 \\
0.4\end{array}$ & $\begin{array}{r}<0.01 \\
0.02 \\
- \\
-\end{array}$ & $\begin{array}{c}80 \pm 2 \\
84 \pm 6 \\
+4 \pm 6 \\
0.5\end{array}$ & $\begin{array}{l}125 \pm 6 \\
101 \pm 5 \\
-24 \pm 7 \\
<0.01\end{array}$ & $\begin{array}{c}+45 \pm 5 \\
+17 \pm 2 \\
-28 \pm 6 \\
<0.01\end{array}$ & $\begin{array}{c}<0.01 \\
<0.01 \\
- \\
-\end{array}$ \\
\hline & \multicolumn{8}{|c|}{$\begin{array}{c}\text { Resistances } \\
\text { dynes seconds } \mathrm{cm}^{-\mathrm{s}}\end{array}$} \\
\hline & \multicolumn{4}{|c|}{ "Total pulmonary" } & \multicolumn{4}{|c|}{ "Pulmonary arteriolar" } \\
\hline & Rest & Exercise & $\begin{array}{c}\text { Response to } \\
\text { exercise }\end{array}$ & $\mathbf{P}$ & Rest & Exercise & $\begin{array}{c}\text { Response to } \\
\text { exercise }\end{array}$ & $\mathbf{P}$ \\
\hline \multirow[t]{4}{*}{$\begin{array}{l}\text { Group A } \\
\text { Group B } \\
\text { Diff. } \\
\text { P }\end{array}$} & $\begin{array}{r}763 \pm 82 \\
565 \pm 112 \\
-198 \pm 140 \\
0.2\end{array}$ & $\begin{array}{r}813 \pm 110 \\
497 \pm 96 \\
-316 \pm 146 \\
0.05\end{array}$ & $\begin{array}{c}+113 \pm 62 \\
-68 \pm 64 \\
-181 \pm 89 \\
0.06\end{array}$ & $\begin{array}{l}0.09 \\
0.3 \\
- \\
-\end{array}$ & $\begin{array}{c}306 \pm 72 \\
364 \pm 100 \\
+58 \pm 123 \\
0.6\end{array}$ & $\begin{array}{c}348 \pm 94 \\
388 \pm 111 \\
+40 \pm 145 \\
0.8\end{array}$ & $\begin{array}{c}+112 \pm 41 \\
-21 \pm 78 \\
-133 \pm 88 \\
0.2\end{array}$ & $\begin{array}{l}0.03 \\
0.8 \\
- \\
-\end{array}$ \\
\hline & \multicolumn{4}{|c|}{$\begin{array}{c}\text { Resistances } \\
\text { dymes seconds } \mathrm{cm}^{-\mathrm{s}}\end{array}$} & & & & \\
\hline & \multicolumn{4}{|c|}{ Total peripheral } & & & & \\
\hline & Rest & Exercise & $\begin{array}{l}\text { Response to } \\
\text { exercise }\end{array}$ & $\mathbf{P}$ & & & & \\
\hline $\begin{array}{l}\text { Group A } \\
\text { Group B } \\
\text { Diff. } \\
\text { P }\end{array}$ & $\begin{array}{c}1,948 \pm 146 \\
1,415 \pm 89 \\
-533 \pm 171 \\
<0.01\end{array}$ & $\begin{array}{c}1,550 \pm 156 \\
1,108 \pm 107 \\
-342 \pm 189 \\
0.03\end{array}$ & $\begin{array}{c}-348 \pm 102 \\
-308 \pm 114 \\
+40 \pm 173 \\
0.8\end{array}$ & $\begin{array}{r}<0.01 \\
0.03 \\
- \\
-\end{array}$ & & & & \\
\hline
\end{tabular}


TABLE III-Continued

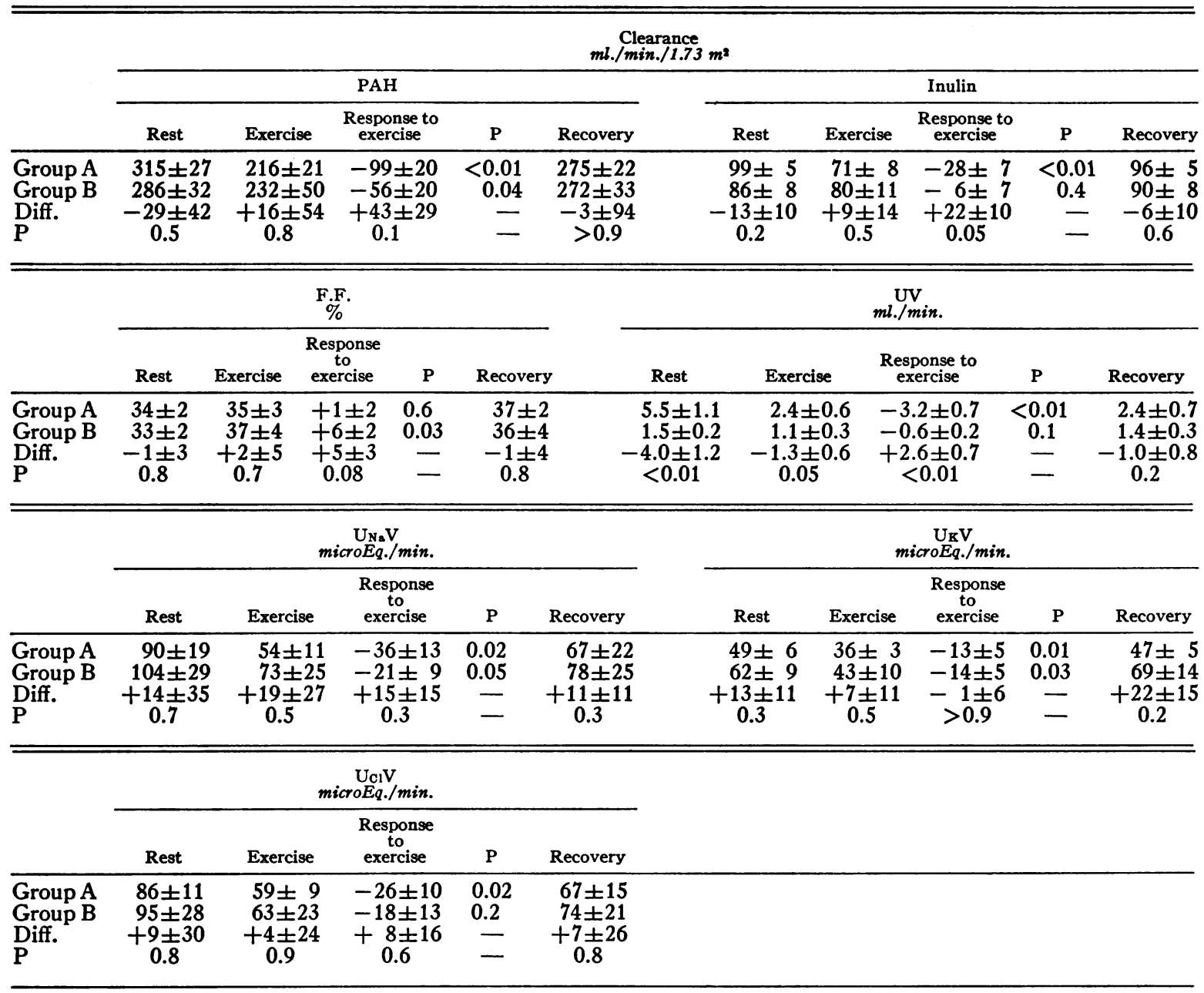

A was within the normal range both at rest and in response to exercise although some individual measurements were slightly below normal. All of the patients except one in Group B had abnormally low arterial oxygen saturations, the average being 78 per cent at rest. There was no significant change in response to exercise (average 76 per cent). The difference in the mean arterial oxygen saturations between the two groups was statistically significant both at rest and during exercise. The mean hematocrit in Group $A$ at rest was within the normal range (average 42 per cent); whereas the mean hematocrit in Group B was abnormally elevated at rest (average 50 per cent), a difference which was statistically significant. The slight increases in hematocrit in both groups during exercise were highly significant statisti- cally, but were not significantly different in the two groups.

\section{Vascular pressures}

Pulmonary arterial pressure. All of the patients in Group A had abnormally elevated mean pulmonary arterial pressures both at rest (average $38 \mathrm{~mm} . \mathrm{Hg}$ ) and during exercise (average $60 \mathrm{~mm}$. $\mathrm{Hg}$ ). Similarly in Group B all except one patient (J. M.) had elevated mean pulmonary arterial pressures at rest (average $39 \mathrm{~mm} . \mathrm{Hg}$ ) and during exercise (average $49 \mathrm{~mm}$. Hg). Although the mean pulmonary arterial pressure during exercise was, on the average, $11 \mathrm{~mm}$. $\mathrm{Hg}$ lower in the patients in Group B than Group A, the variation in the individual data was so great that this 
difference was not statistically significant. In response to exercise, the mean pulmonary arterial pressure rose $22 \mathrm{~mm}$. $\mathrm{Hg}$ in Group A and only 10 $\mathrm{mm} . \mathrm{Hg}$ in Group B; this difference was highly significant both physiologically and statistically.

"Pulmonary capillary" pressure. At rest the mean "pulmonary capillary" (PC) pressure in all but one patient (A. J.) in Group A was above normal (average $22 \mathrm{~mm}$. $\mathrm{Hg}$ ). In Group B five of the seven patients in whom the mean "PC" pressure was measured had values that were above or near the upper limits of normal (average 14 $\mathrm{mm}$. $\mathrm{Hg}$ ). The difference between the groups, averaging $8 \mathrm{~mm}$. $\mathrm{Hg}$, was highly significant. In response to exercise the mean "PC" pressure rose on the average by $14 \mathrm{~mm}$. $\mathrm{Hg}$ (to $36 \mathrm{~mm} . \mathrm{Hg}$ ) in Group A, but by only $2 \mathrm{~mm}$. $\mathrm{Hg}$ (to $17 \mathrm{~mm}$. $\mathrm{Hg}$ ) in Group B. This difference was also highly significant.

\section{Right ventricular end diastolic pressure}

In nine of the fifteen patients in Group A the right ventricular end diastolic (R.V.D.) pressure was elevated at rest (the average for the entire group being $9 \mathrm{~mm}$. $\mathrm{Hg}$ ) ; in five of the patients in Group $B$ it was likewise elevated (average $11 \mathrm{~mm} . \mathrm{Hg}$ ). During exercise the average R.V.D. pressure rose by $15 \mathrm{~mm}$. $\mathrm{Hg}$ in Group $\mathrm{A}$ and $19 \mathrm{~mm}$. $\mathrm{Hg}$ in Group B. The average increases in R.V.D. pressure in response to exercise were approximately equal in the two groups, and the difference between them was not statistically significant.

Arterial pressure. The mean arterial pressure at rest was within the normal range for both Group A (average $95 \mathrm{~mm}$. $\mathrm{Hg}$ ) and Group B (average $100 \mathrm{~mm}$. $\mathrm{Hg}$ ). In both groups the mean arterial pressure showed a highly significant increase during exercise. However, the response of the arterial pressure to exercise was not significantly different between the two groups.

Heart rate. The heart rate at rest averaged 80 beats per minute in Group $A$ and 84 beats per minute in Group B. However, the increase in heart rate in response to exercise was, on the average, 28 beats per minute greater in Group A than in Group B. This highly significant difference in response to exercise may have resulted from inadequate digitalization and stabilization of the heart rate in several patients in Group A who had auricular fibrillation.

\section{Vascular resistances (in units of dynes seconds $\mathrm{cm}^{-5}$ )}

Vascular resistances were calculated according to formulae previously discussed and defined (9). The so-called "total pulmonary" resistance, which actually includes the total resistance opposing the right ventricle, was elevated at rest in Group A (average 763 units) and in Group B (average 565 units); the difference was not statistically significant. In response to exercise total pulmonary resistance rose in Group A (113 units) while it decreased on the average 68 units in Group B. This difference proved to be just significant statistically.

The "pulmonary arteriolar" resistance, actually the total pulmonary vascular resistance, was calculated to be increased at rest in both Group A (average 306 units) and Group B (average 364 units). This difference was not statistically significant. However, there was an average increase of 112 units in "pulmonary arteriolar" resistance in response to exercise in nine patients in Group A while there was an average decrease of 21 units in Group B. The difference in the two groups in response to exercise was not significant by statistical analysis.

Since the cardiac output was significantly lower in the Group A patients than in Group B, their mean arterial pressures were maintained at equal levels as a result of a greater total peripheral resistance (1,948 units compared with 1,415 units in Group B). This difference was highly significant statistically. However, in response to exercise the decrease in total systemic resistance in the two groups was not significantly different.

\section{Renal responses}

At rest. The renal plasma flow was abnormally low in all the patients in both Group A (mean $315 \mathrm{ml}$. per min. per $1.73 \mathrm{~m}^{2}$ ) and Group B (mean $286 \mathrm{ml}$. per min. per $1.73 \mathrm{~m}^{2}$ ). Glomerular filtration rates, measured in thirteen of the Group A patients, averaged $99 \mathrm{~mm}$. per min. per $1.73 \mathrm{~m}^{2}$, which is abnormally low. Seven of these patients had low glomerular filtration rates, and six were within the range of normals. In contrast, six of the seven patients in Group $B$ in whom this function was measured had abnormally low values, the average for the group being 86 
ml. per min. per $1.73 \mathrm{~m}^{2}$ (9).4 Although the average renal plasma flow and glomerular filtration rate measurements tended to be lower in Group B than Group A, the differences were not statistically significant. Inasmuch as the dietary intake of salt was not uniformly controlled in the patients in these two groups, the differences in water and electrolyte excretion at rest in relation to the differences between cardiovascular and renal hemodynamic measurements in the two groups could not be evaluated.

During exercise. During exercise the averages of renal plasma flow and glomerular filtration rate in Group A were $216 \mathrm{ml}$. per min. per $1.73 \mathrm{~m}^{2}$ and $71 \mathrm{ml}$. per $\min$. per $1.73 \mathrm{~m}^{2}$, respectively. Thus, in response to exercise in Group A the renal plasma flow decreased on the average $99 \mathrm{ml}$. per min. per $1.73 \mathrm{~m}^{2}$ or 31 per cent and the glomerular filtration rate fell on the average $28 \mathrm{ml}$. per min. per $1.73 \mathrm{~m}^{2}$ or 28 per cent. These decreases during exercise in Group A were highly significant statistically. In Group B during exercise the averages in the renal plasma flow and glomerular filtration rate dropped to $232 \mathrm{ml}$. per min. per $1.73 \mathrm{~m}^{2}$ and $80 \mathrm{ml}$. per min. per $1.73 \mathrm{~m}^{2}$, respectively. Thus, the mean decreases in renal plasma flow and glomerular filtration rate in response to exercise in the patients in Group B were considerably less than in Group A, the average renal plasma flow decreasing by $56 \mathrm{ml}$. per min. per $1.73 \mathrm{~m}^{2}$ or 20 per cent and the glomerular filtration rate by $6 \mathrm{ml}$. per min. per $1.73 \mathrm{~m}^{2}$ or 7 per cent. Although the fall in the renal plasma flow in response to exercise in Group A was not significantly greater than that in Group $B$, the decrease in glomerular filtration rate in Group $A$ was significantly greater than the relatively unchanged response in Group $B$. The filtration fraction, elevated at rest in both Group A and B (averaging 34 and 33 per cent, respectively), increased only slightly during exercise in Group A but significantly in Group B. This latter change was consistent with the disproportionately greater

4 The values for renal plasma flow and glomerular filtration rate are lower than those reported by Fishman, Maxwell, Crowder, and Morales (9) in a similar group of patients with chronic pulmonary disease without congestive failure. These differences may be due to a greater incidence of clinically unrecognizable renal disease or arteriosclerotic changes of the kidneys in our group. reduction in the renal plasma flow than in the glomerular filtration rate during exercise.

In the Group A patients during exercise the decreases in the excretion of water ( 56 per cent), sodium ( 40 per cent), chloride (30 per cent), and potassium (27 per cent) were highly significant. In general, these reductions tended to parallel the decreases in the renal plasma flow and glomerular filtration rate induced by exercise. In two patients (A. J., F. A.) the renal hemodynamic measurements did not change but there was nevertheless a decrease in electrolyte excretion during exercise. In another patient J. C. there were significant changes in the renal hemodynamic measurements unassociated with changes in rate of electrolyte excretion. Two patients in Group A (C. J., C. S.) showed a significant decrease neither in renal hemodynamic function nor in excretion of electrolytes during exercise.

On the average, the Group B patients had less marked decreases in the excretion of water, sodium, chloride, and potassium during exercise. Of these measurements the reductions in sodium and potassium excretion were statistically significant. Only two of the patients (J. M., M. M.) had falls in both renal clearances (PAH and inulin) and in excretion of electrolytes and water during exercise. One patient (D. K.) showed no change in either renal clearances or excretory function. Another patient (M. G.) had a fall in the excretion of sodium, chloride, and potassium despite no reduction in the renal clearance measurements. Finally another patient (W. D.) showed a decrease in renal clearances without any appreciable change in the excretion of sodium and chloride. The magnitude of the decreases in the excretion of sodium, chloride, and potassium in response to exercise was not significantly different in Group A and Group B.

Recovery from exercise. In six of ten patients in Group A in whom exercise had produced depression of renal hemodynamic measurements, these functions had returned to the pre-exercise control values within 20 to 30 minutes of the recovery period. In the other four patients persistently low values of the glomerular filtration rate and/or renal plasma flow were observed during the entire recovery period of measurement which lasted for 26 to 45 minutes after the completion of exercise. The excretion of electrolytes, 
and usually of water, remained at a low level throughout the recovery phase not only in those patients who had a persistent lowering of the renal plasma flow and glomerular filtration rate but also in patients in whom the latter functions had shown prompt recovery. This persistent decrease in the excretion of water and electrolytes in most of the patients, therefore, appeared to be independent of changes in the renal clearance measurements (Figure 1).

In Group B the renal plasma flow and/or glomerular filtration rate had returned to the control value in all the patients in 20 to 30 minutes after the exercise period. However, the persistent reduction in the excretion of sodium and chloride in four patients (M. A., W. H., J. M., M. G.) during the recovery phase again suggested that the retention of these electrolytes was independent of any measurable alterations in renal plasma flow or glomerular filtration rate. Except in a few patients the excretion of water returned to the preexercise control level during the recovery period. Although the excretion of electrolytes tended to return more promptly to the control values in the Group $B$ patients during the recovery phase, statistical analysis of the mean data showed no significant difference between Group A and Group B.

Two patients with so-called "high output" failure (M. S. with hypertensive cardiovascular disease and acute posthemorrhagic anemia, and H. H. with large systemic arteriovenous fistula) who were not included in the statistical analysis, also had abnormally low renal plasma flows and glomerular filtration rates at rest. Patient $\mathrm{H}$. $\mathrm{H}$. had the higher cardiac output and the more depressed renal plasma flow at rest. Both patients during exercise showed increases in intracardiac and pulmonary arterial pressures but had normal increases in cardiac output. With these cardiohemodynamic responses during exercise, however, there was slight or no change in the renal plasma flow and glomerular filtration rate. Patient M. S., who showed no significant change in either glomerular filtration rate or renal plasma flow during exercise, had a marked and persistent decrease in the excretion of water and sodium after exercise. Patient $\mathrm{H}$. $\mathrm{H}$. showed a transitory decrease in the excretion of sodium during exercise, but prior evidence of marked retention of sodium at
J.Di.8 38 - RH.D. MI., A.F. IT

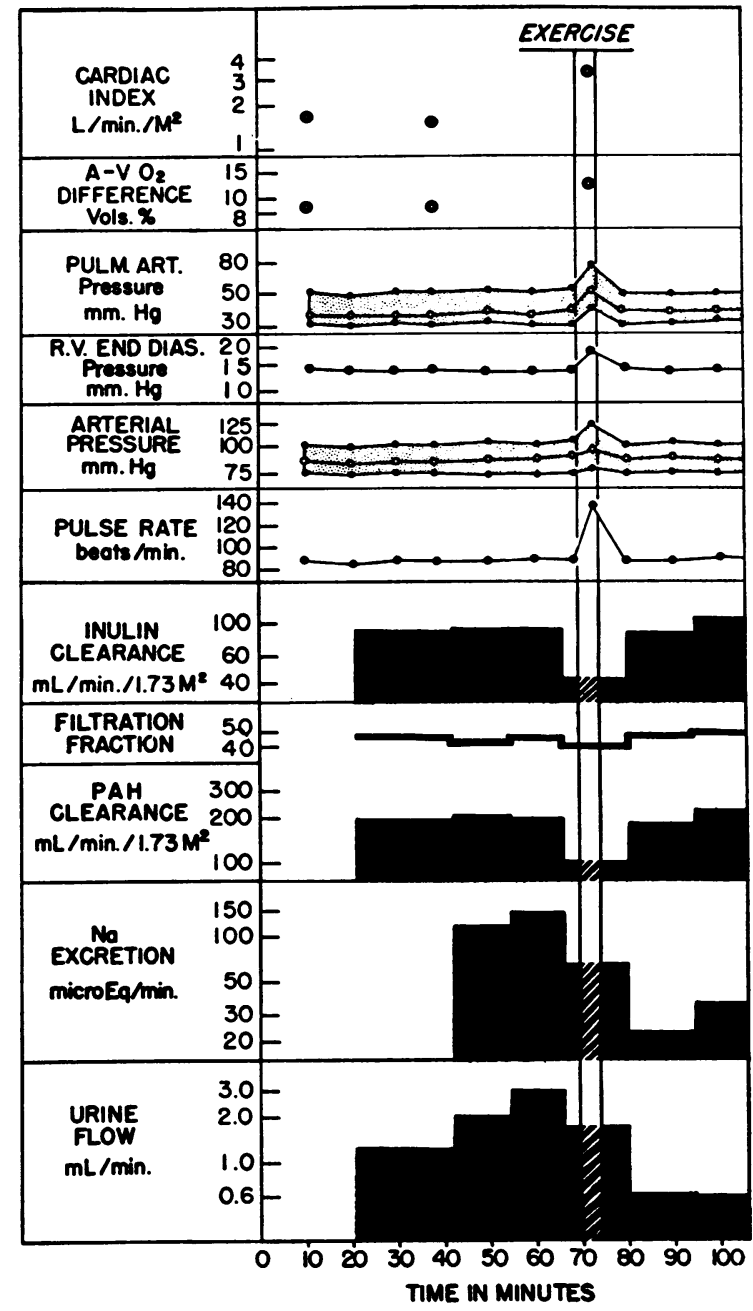

Fig. 1. Chart of the Cardiovascular and Renal Measurements at Rest and during Exercise in the Supine Position in Patient J. Di. with Severe Mitral INSUFFICIENCY

rest in this patient made further reductions in the excretion of urinary sodium difficult to evaluate accurately.

\section{DISCUSSION}

In this group of patients no significant correlation was found between the cardiac output and the renal blood flow and glomerular filtration rate at rest. Statistical analysis of a larger group of thirty-eight patients with "low output" congestive failure studied in this laboratory likewise showed no absolute relationship between these functions. Also there was no consistent correlation between the degrees of abnormality in renal plasma flow 
and in glomerular filtration rate and the mixed arteriovenous oxygen difference, right ventricular end diastolic, pulmonary arterial and "pulmonary capillary" pressures.

In all the patients with pulmonary disease the renal plasma flows at rest were decreased despite cardiac outputs and arteriovenous oxygen differences which were within the normal range. Although there was a significant difference in the level of the cardiac indices at rest between Group A and B, the renal plasma flows and glomerular filtration rates were not significantly different in the two groups. These reductions in renal plasma flow and glomerular filtration rate in these patients with chronic pulmonary disease were not directly related to the cardiac output, arteriovenous oxygen difference, arterial oxygen saturation, hematocrit, intracardiac or pulmonary vascular pressures.

Some patients with clinical peripheral edema had normal glomerular filtration rates at rest. Other patients without peripheral edema who were not restricting their dietary intake of salt had abnormally low cardiac outputs and glomerular filtration rates. A more detailed study concerning the relationship of cardiovascular and renal hemodynamic functions to the renal excretion of salt and water in patients with congestive failure has been done and will be presented in a separate report (10). The presence or absence of peripheral edema in these patients could not be predicted from the changes in their cardiodynamics or renal responses during or after exercise.

In the present study on the cardiac and renal responses to exercise the effects of postural factors have been eliminated. Numerous investigators have reported that exercise in normal subjects causes decreases in renal plasma flow and, to a lesser extent, in the glomerular filtration rate and that these falls are greater in the upright than in the supine position (1-5). The degree of depression of the renal clearance measurements has also been related roughly to the severity of the exercise (3). Merrill and Cargill (11) have found that the reduction in the renal plasma flow and glomerular filtration rate during exercise is greater in supine patients with congestive heart failure than in normal subjects. This greater decrease in the renal circulation has been considered to be related to an inadequate cardiac out- put in the cardiac patients during exercise. Exercise in the upright position in normal subjects and also in patients with congestive failure has been shown to produce persistent decreases in the excretion of water, sodium and usually of chloride (1-6). In general these persistent decreases appeared to be independent of changes in the glomerular filtration rate and therefore were attributed to increased tubular reabsorption on a hormonal basis. Sinclair-Smith, Kattus, Genest, and Newman suggest that the persistence of the depression of sodium excretion after exercising in the upright position is about the same in patients with congestive heart failure as in normal subjects (6).

In normal subjects exercising in the supine position for one-half hour by alternate straight legraising (resulting in a 3 to 9 fold increase in oxygen consumption), Hatcher, Huckabee, Epstein, and Wilkins (7) have observed a significant reduction in renal plasma flow and only a slight or no decrease in the glomerular filtration rate. The renal plasma flow usually remained depressed for the first 10 to 15 -minute period after the completion of exercise and returned to the control values by the second or third recovery periods. A reduction in sodium excretion was sometimes observed during exercise, and this was usually associated with a decrease in glomerular filtration rate. Moreover, when the glomerular filtration rate and renal plasma flow recovered to control values following exercise, there was a prompt return of sodium excretion to the initial control levels. These studies suggest that although exercise may cause a decrease in the renal plasma flow and, to a lesser degree, glomerular filtration rate, exercise per se does not cause a persistent reduction in sodium and water excretion in normal healthy subjects. They also suggest that the greater tubular reabsorption of sodium and water and the larger decreases in glomerular filtration rate and renal plasma flow in patients exercising in the upright position is for the most part related to the posture itself. This view is supported by other studies $(3,4,12-14)$ which have shown that the upright posture alone in normal subjects causes an increased renal tubular reabsorption of sodium and water and a decrease in renal plasma flow associated with slight or no fall in glomerular filtration rate. 
Since in the present study the influence of postural factors on salt and water excretion have been eliminated, the renal responses to exercise may be compared to those observed by Hatcher, Huckabee, Epstein, and Wilkins. In patients with "low output" types of congestive failure the reductions in renal plasma flow and glomerular filtration rate were greater during exercise than in normal subjects. However, the renal hemodynamic responses during exercise in patients with chronic pulmonary disease resembled those of the normal subjects in showing a slight or insignificant reduction in the glomerular filtration rate. In general the return of the renal hemodynamic measurements to control values after a fall in response to exercise, in patients with any form of congestive failure, required a longer period of time than in normal subjects. Furthermore, the patients with "low output" forms of heart disease and those with chronic pulmonary disease had a more definite and greater decrease in sodium excretion during exercise than normal subjects. In the patients with "low output" congestive failure the reduction in sodium excretion usually paralleled the fall in the glomerular filtration rate while in the patients with chronic pulmonary disease, who had comparable changes in sodium excretion, this alteration in glomerular filtration rate was not so frequently observed. (Unfortunately, in two patients with the most severe cor pulmonale and congestive failure no measurements of the renal hemodynamics could be obtained during exercise.) A majority of patients in congestive failure had a more persistent reduction in sodium excretion after exercise than did the normal subjects. This persistent decrease in sodium excretion following exercise usually appeared to be independent of changes in glomerular filtration rate and was attributed to increased renal tubular reabsorption. The degree and duration of depression of the renal blood flow and glomerular filtration rate during and following exercise was not necessarily related to the degree and duration of the changes in cardiac output, mixed arteriovenous oxygen difference, arterial oxygen saturation, and the intracardiac, pulmonary arterial and renal venous pressures. Calculation of correlation coefficients also failed to reveal any correlation between the variation in the sodium excretion and the various cardiovascular responses.
The mechanisms for the increased tubular reabsorption of sodium during and after exercise were not apparent from this study.

Werkö, Varnauskas, Eliasch, Ek, Bucht, Thomasson, and Bergström (15) have reported in clinical group IV patients with mitral stenosis and right heart failure with elevated peripheral venous pressure that although exercise in the supine position produced the usual slight lowering of the glomerular filtration rate and retention of sodium, it did not produce a further decrease of the renal plasma flow as it usually did in similar patients not in congestive failure. Since their patients with congestive failure also differed from the compensated group by failing to increase their cardiac outputs with exercise, the authors concluded that maximal depression in renal plasma flow had already occurred. They explained this as being "mainly because of the low cardiac output which did not allow any 'superfluous' renal blood flow." From these observations they also concluded that the decrease in renal blood flow during exercise is not necessarily related to further elevations of venous pressure in the kidney. In contrast, in our patients with "low output" failure and elevated peripheral venous pressure the low resting cardiac outputs were still capable of being increased in response to exercise while the low renal plasma flows showed the usual further reductions. This difference in responsiveness might be explained by the fact that in the study by Werkö and associates the amount of exercise performed caused an average elevation of about 70 per cent in oxygen consumption, while the more strenuous exercise employed in the present study resulted in about 150 per cent increase in oxygen consumption.

\section{SUMMARY}

Studies of renal functional responses to exercise in cardiac patients in the supine position showed (in contrast to those in normal subjects who have a transitory fall in renal plasma flow and a slight or no change in the glomerular filtration rate and excretion of sodium):

1. That Group A patients (with severe and usually decompensated valvular or non-valvular heart disease) had significant reductions in the renal plasma flow (31 per cent), glomerular fil- 
tration rate (28 per cent), and excretion of sodium (40 per cent).

2. That Group B patients (with severe chronic pulmonary disease with or without congestive failure) had a fall in renal plasma flow (20 per cent), and a slight decrease in glomerular filtration rate (7 per cent), associated with a significant reduction in the excretion of sodium (20 per cent).

3 . That the significantly greater reduction in glomerular filtration rate in Group A than in Group B patients was not associated with a greater reduction in sodium excretion.

4. That in the recovery phase there was a more rapid return of the renal hemodynamic measurements than of the excretion of sodium to the preexercise control values. This dissociation suggests that the retention of sodium was independent of any measurable alteration in glomerular filtration rate and probably resulted from a change in renal tubular activity.

5. That neither the magnitude nor the duration of the depression of the excretion of sodium was consistently related to the degree or duration of reduction in the glomerular filtration rate.

6 . That no correlation could be determined between the degree or duration of reductions in renal blood flow, glomerular filtration rate, or the excretion of sodium and the degree of the cardiovascular responses.

\section{REFERENCES}

1. Eggleton, M. G., The effect of exercise on chloride excretion in man during water diuresis and tea diuresis. J. Physiol., 1943, 102, 140.

2. Barclay, J. A., Cooke, W. T., Kenney, R. A., and Nutt, M. E., The effects of water diuresis and exercise on the volume and composition of the urine. Am. J. Physiol., 1947, 148, 327.

3. White, H. L., and Rolf, D., Effects of exercise and of some other influences on the renal circulation in man. Am. J. Physiol., 1948, 152, 505.

4. Kattus, A. A., Sinclair-Smith, B., Genest, J., and Newman, E. V., The effect of exercise on the renal mechanism of electrolyte excretion in normal subjects. Bull. Johns Hopkins Hosp., 1949, 84, 344.
5. Chapman, C. B., Henschel, A., Minckler, J., Forsgren, A., and Keys, A., The effect of exercise on renal plasma flow in normal male subjects. J. Clin. Invest., 1948, 27, 639.

6. Sinclair-Smith, B., Kattus, A. A., Genest, J., and Newman, E. V., The renal mechanism of electrolyte excretion and the metabolic balances of electrolytes and nitrogen in congestive cardiac failure; the effects of exercise, rest and aminophyllin. Bull. Johns Hopkins Hosp., 1949, 84, 369.

7. Hatcher, J. D., Huckabee, W. E., Epstein, F. H., and Wilkins, R. W., The effects of exercise on the renal hemodynamics and electrolyte excretion in normal supine subjects. To be published.

8. Judson, W. E., Hatcher, J. D., Hollander, W., and Halperin, M. H., The effects of mitral valvuloplasty on cardiovascular and renal function at rest and during exercise. J. Clin. Invest., 1955, 34, 1297.

9. Fishman, A. P., Maxwell, M. H., Crowder, C. H., and Morales, P., Kidney function in cor pulmonale. Particular consideration of changes in renal hemodynamics and sodium excretion during variation in level of oxygenation. Circulation, 1951, 3, 703.

10. Hollander, W., Judson, W. E., Halperin, M. H., and Freidman, I., The effects of acute salt loading on the cardiorenal hemodynamics and the excretion of electrolytes and water in patients with congestive heart failure. To be published.

11. Merrill, A. J., and Cargill, W. H., The effect of exercise on the renal plasma flow and filtration rate of normal and cardiac subjects. J. Clin. Invest., 1948, 27, 272.

12. Epstein, F. H., Goodyer, A. V. N., Lawrason, F. D., and Relman, A. S., Studies of the antidiuresis of quiet standing: The importance of changes in plasma volume and glomerular filtration rate. $\mathrm{J}$. Clin. Invest., 1951, 30, 63.

13. Brun, C., Knudsen, E. O. E., and Raaschou, F., The influence of posture on the kidney function. I. The fall of diuresis in the erect posture. Acta med. Scandinav., 1945, 122, 315.

14. Brun, C., Knudsen, E. O. E., and Raaschou, F., The influence of posture on the kidney function. II. Glomerular dynamics in the passive erect posture. Acta med. Scandinav., 1945, 122, 332.

15. Werkö, L., Varnauskas, E., Eliasch, H., Ek, J., Bucht, H., Thomasson, B., and Bergström, J., Studies on the renal circulation and renal function in mitral valvular disease. I. Effect of exercise. Circulation, 1954, 9, 687. 